\title{
Pediatric NAFLD: lessons from the gut
}

\author{
Laura Iogna Prat, Emmanuel A. Tsochatzis
}

UCL Institute for Liver and Digestive Health, Royal Free Hospital, University College of London, London, UK

Correspondence to: Emmanuel A. Tsochatzis. UCL Institute for Liver and Digestive Health, Royal Free Hospital, University College of London, Pond Street, NW3 2QG, London, UK. Email: e.tsochatzis@ucl.ac.uk.

Provenance and Peer Review: This article was commissioned by the editorial office, Hepatobiliary Surgery and Nutrition. The article did not undergo external peer review.

Comment on: Schwimmer JB, Johnson JS, Angeles JE, et al. Microbiome signatures associated with steatohepatitis and moderate to severe fibrosis in children with nonalcoholic fatty liver disease. Gastroenterology 2019;157:1109-22.

Submitted Jan 03, 2020. Accepted for publication Jan 28, 2020.

doi: 10.21037/hbsn.2020.01.06

View this article at: http://dx.doi.org/10.21037/hbsn.2020.01.06

Over the last decade numerous studies have focused on characterizing the human microbiome; particular attention has been paid to understanding the complex interplay between gut, its microbiota and the liver (the so called "gutliver axis"). The disruption of the gut-liver axis is involved in the development of several metabolic alterations such as obesity and non-alcoholic fatty liver disease (NAFLD) (1).

Among liver diseases, NAFLD constitutes a worldwide epidemic, affecting around $25 \%$ of adults and $8 \%$ of children in western countries (2). A consistent body of evidence has emerged in pre-clinical models regarding the role of microbiota in the development of NAFLD: for example, dysbiosis can enhance the response of mice models to high-fat diet and this growth promoting phenotype can be transferred to germ-free hosts via colonisation with intestinal microbiota. Furthermore, disruption in the gut microbiota by low dose antibiotics in the early life is sufficient to induce sustained effects on body composition in the long term $(3,4)$. In human studies, the association between gut dysbiosis and development of metabolic alterations is mainly supported by crosssectional data, therefore a causative link has not been conclusively proven yet. Nonetheless, over the last years studies have been consistent in describing a lower species richness (the so called $\alpha$-diversity) in the microbiota of patients with obesity and/or NAFLD compared to healthy subjects both in children and in adults (5-7). Some studies have also identified shifts towards a higher representation of specific phyla in people with NAFLD but results have been discordant in some cases. For example, Turnbaugh et al. found a lower proportion of Bacteroidetes and a higher proportion of Actinobacteria in adult obese individuals compared to their lean twin pairs (7); similarly, Mouzaki et al. found a negative association between Bacteroidetes and non-alcoholic steatohepatitis (NASH) which was independent from BMI and dietary fat intake (8). On the contrary other studies have reported an increase in Bacteroidetes in obese and NASH patients alongside a decrease in Firmicutes compared to healthy controls (5). These apparently conflicting results can be attributed to the relatively small sample size of the populations studied, their different characteristics (particularly in terms of geography and diet) and different techniques adopted. Regardless of the specific composition of the microbiota, it has been described that dysbiosis not only characterizes the presence of NAFLD but shows particular features according to the disease severity. Boursier et al. studied the microbiota among adults presenting the whole spectrum of NAFLD, from simple steatosis (NAFL) to NASH with various stages of fibrosis, showing that Bacteroides, Prevotella and Ruminococcus had different patterns of abundance in these patients. Importantly, stratification according to their abundance identified three subgroups of patients with increasing severity of NAFLD lesions. Interestingly, Bacteroides abundance was independently associated with NASH and Ruminococcus with significant fibrosis independently from the traditional metabolic risk factors. Additionally, based on imputed metagenomics profile, a shift towards pathways related to carbohydrates, lipid and amino acid metabolism was highlighted in patients with more severe disease (9). 
Although all these studies improved our understanding of the pathophysiology of NAFLD, they face the complexity of microbiota inter- and intra-individual variability. It is important to keep in mind that microbiota originates in our early life and that they represent a host "fingerprint", potentially predisposing to weight gain, and the development and progression of NAFLD. Childhood constitutes therefore a precious window to study how microbial diversity impacts on energy harvest and utilization and consequently the development of liver disease. Kalliomäki et al. compared groups of children over time and demonstrated that those who became overweight by the age of 7 had a lower level of Bifidobacteria and a higher level of Staphylococcus aureus as infants compared to those who kept a normal weight (10). Another study on overweight adolescents demonstrated that microbiota confers significant predisposition to develop obesity and affects the effectiveness of life-style interventions once obesity is established (11). Additionally, intestinal permeability is impaired in children aged 5-8 years old with early stage NAFLD compared to controls, suggesting that dysbiosis and altered permeability of the intestinal barrier can be detected before full-blown signs of liver disease develop (12). Far from having completely characterized the causative link between gut microbes and fatty liver, some clinical trials have attempted so far to manipulate microbiota in order to improve clinical and biochemical signs in children with NAFLD. Alisi et al. carried out a randomized clinical trial in 44 children with biopsy proven NAFLD using VSL\#3, a mixture of 8 probiotic strains, administered for 4 months. At the end of the study a significant improvement of fatty liver assessed by ultrasound and a significant decrease in BMI were detected in the treatment group compared to placebo (13). A mixture of 4 probiotic strains was used by Famouri et al. who carried out a RCT on 64 obese children aged 10-18 who presented NAFLD diagnosed by ultrasound. In this study, a significant decrease in liver enzymes, cholesterol levels and waist circumference was detected in the treatment group compared to placebo. Moreover, among children in the treatment group the rate of normalization of the ultrasound was higher than in the placebo group (14). Overall these results, although promising, are biased by the overlap of life-style interventions which were concurrently applied with treatment and by the use of surrogate outcomes to define response to treatment.

Considering the state of the art, the recently published study by Schwimmer et al. provides important information and novel high-quality data, as it involves a high number of children with biopsy proven NAFLD and a well-matched control group and relies on cutting edge technology for the microbiome genome sequencing and function (15). The study included 87 children with biopsy proven NAFLD and 37 children with obesity but no NAFLD. Magnetic resonance spectroscopy was used for the exclusion of NAFLD in controls. The microbiome composition and function was assessed using 16s rRNA amplicon sequencing and metagenomic shotgun sequencing. The study accurately described taxonomic differences in microbiota composition between children with NAFLD, NASH and controls both at the phyla and the genus level. There were various important findings from this study. Pediatric NAFLD was associated with a decline in $\alpha$-diversity, with no evidence of lower metabolic potential despite lower diversity. There was also greater person-to-person variability in the composition of the microbiome in children with NAFLD. A high abundance of Prevotella Copri was associated with lower in $\alpha$-diversity and more severe fibrosis. Whether this loss of diversity and abundance of Prevotella Copri is cause or effect of NAFLD remains to be proven.

Moreover, different metabolic pathways have been brought into light with an enhancement of proinflammatory patterns characterizing the more severe stages of fibrosis. Genes influencing lipopolysaccharide biosynthesis and genes associated with flagellar assembly had greater relative abundance in NAFLD versus controls and in NASH versus NAFLD or moderate-tosevere versus mild fibrosis respectively. Predictive models using classification and regression trees including data derived from gene set enrichment analysis together with biochemical data have demonstrated excellent diagnostic accuracy to detect the presence of NASH and moderate to severe fibrosis in this population with area under the receiver operating characteristics (AUROCs) over 0.90.

Weaknesses of the study include the relatively small sample size, the wide range of the included pediatric population (8-17 years old) and the absence of a validation cohort. Moreover, association does not prove causality and therefore it is unclear if the observed changes in the microbiome are a cause or an effect of NAFLD.

Nevertheless, these results constitute a step forward to a better understanding of the role of the microbiome in patients with NAFLD, and a particular strength was the inclusion of patients at different stages of disease. The good accuracy of the regression tree models including microbiota features in detecting NASH and moderate-to severe fibrosis are encouraging and will hopefully pave the 
way for developing new diagnostic tests for children with NAFLD. Further studies on the composition and potential manipulation of the microbiome in children and adults are required to enhance our knowledge and potentially offer therapeutic options for NAFLD and NASH.

\section{Acknowledgments}

Funding: None.

\section{Footnote}

Conflicts of Interest: Both authors have completed the ICMJE uniform disclosure form (available at http://dx.doi. org/10.21037/hbsn.2020.01.06). The authors have no conflicts of interest to declare.

Ethical Statement: The authors are accountable for all aspects of the work in ensuring that questions related to the accuracy or integrity of any part of the work are appropriately investigated and resolved.

Open Access Statement: This is an Open Access article distributed in accordance with the Creative Commons Attribution-NonCommercial-NoDerivs 4.0 International License (CC BY-NC-ND 4.0), which permits the noncommercial replication and distribution of the article with the strict proviso that no changes or edits are made and the original work is properly cited (including links to both the formal publication through the relevant DOI and the license). See: https://creativecommons.org/licenses/by-nc$\mathrm{nd} / 4.0 /$.

\section{References}

1. Albillos A, Gottardi A, Rescigno M. The gut-liver axis in liver disease: pathophysiological basis for therapy. J Hepatol 2020;72:558-77.

2. Tsochatzis EA, Newsome PN. Non-alcoholic fatty liver disease and the interface between primary and secondary care. Lancet Gastroenterol Hepatol 2018;3:509-17.

3. Le Roy T, Llopis M, Lepage P, et al. Intestinal microbiota determines development of non-alcoholic fatty liver disease in mice. Gut 2013;62:1787-94.

4. Cox LM, Yamanishi S, Sohn J, et al. Altering the intestinal microbiota during a critical developmental window has lasting metabolic consequences. Cell 2014;158:705-21.
5. Zhu L, Baker SS, Gill C, et al. Characterization of gut microbiomes in nonalcoholic steatohepatitis (NASH) patients: a connection between endogenous alcohol and NASH. Hepatology 2013;57:601-9.

6. Del Chierico F, Nobili V, Vernocchi P, et al. Gut microbiota profiling of pediatric nonalcoholic fatty liver disease and obese patients unveiled by an integrated metaomics-based approach. Hepatology 2017;65:451-64.

7. Turnbaugh PJ, Hamady M, Yatsunenko T, et al. A core gut microbiome in obese and lean twins. Nature 2009;457:480-4.

8. Mouzaki M, Comelli EM, Arendt BM, et al. Intestinal microbiota in patients with nonalcoholic fatty liver disease. Hepatology 2013;58:120-7.

9. Boursier J, Mueller O, Barret M, et al. The severity of nonalcoholic fatty liver disease is associated with gut dysbiosis and shift in the metabolic function of the gut microbiota. Hepatology 2016;63:764-75.

10. Kalliomäki M, Collado MC, Salminen S, et al. Early differences in fecal microbiota composition in children may predict overweight. Am J Clin Nutr 2008;87:534-8.

11. Santacruz A, Marcos A, Wärnberg J, et al. Interplay between weight loss and gut microbiota composition in overweight adolescents. Obesity (Silver Spring) 2009;17:1906-15.

12. Nier A, Engstler AJ, Maier IB, et al. Markers of intestinal permeability are already altered in early stages of nonalcoholic fatty liver disease: studies in children. PLoS One 2017; 12:e0183282.

13. Alisi A, Bedogni G, Baviera G, et al. Randomised clinical trial: the beneficial effects of VSL\#3 in obese children with non-alcoholic steatohepatitis. Aliment Pharmacol Ther 2014;39:1276-85.

14. Famouri F, Shariat Z, Hashemipour M, et al. Effects of probiotics on nonalcoholic fatty liver disease in obese children and adolescents. J Pediatr Gastroenterol Nutr 2017;64:413-7.

15. Schwimmer JB, Johnson JS, Angeles JE, et al. Microbiome signatures associated with steatohepatitis and moderate to severe fibrosis in children with nonalcoholic fatty liver disease. Gastroenterology 2019;157:1109-22.

Cite this article as: Iogna Prat L, Tsochatzis EA. Pediatric NAFLD: lessons from the gut. HepatoBiliary Surg Nutr 2020;9(4):534-536. doi: 10.21037/hbsn.2020.01.06 